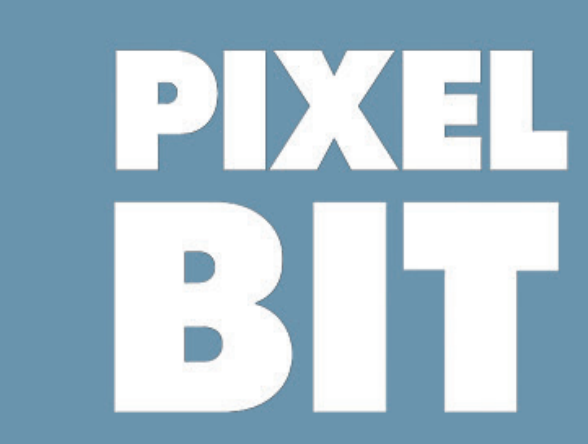

Revista de Medios y Educación

№ 58 MAYO 2020 CUATRIMESTRAL e-ISSN:2171-7966 ISSN:1133-8482
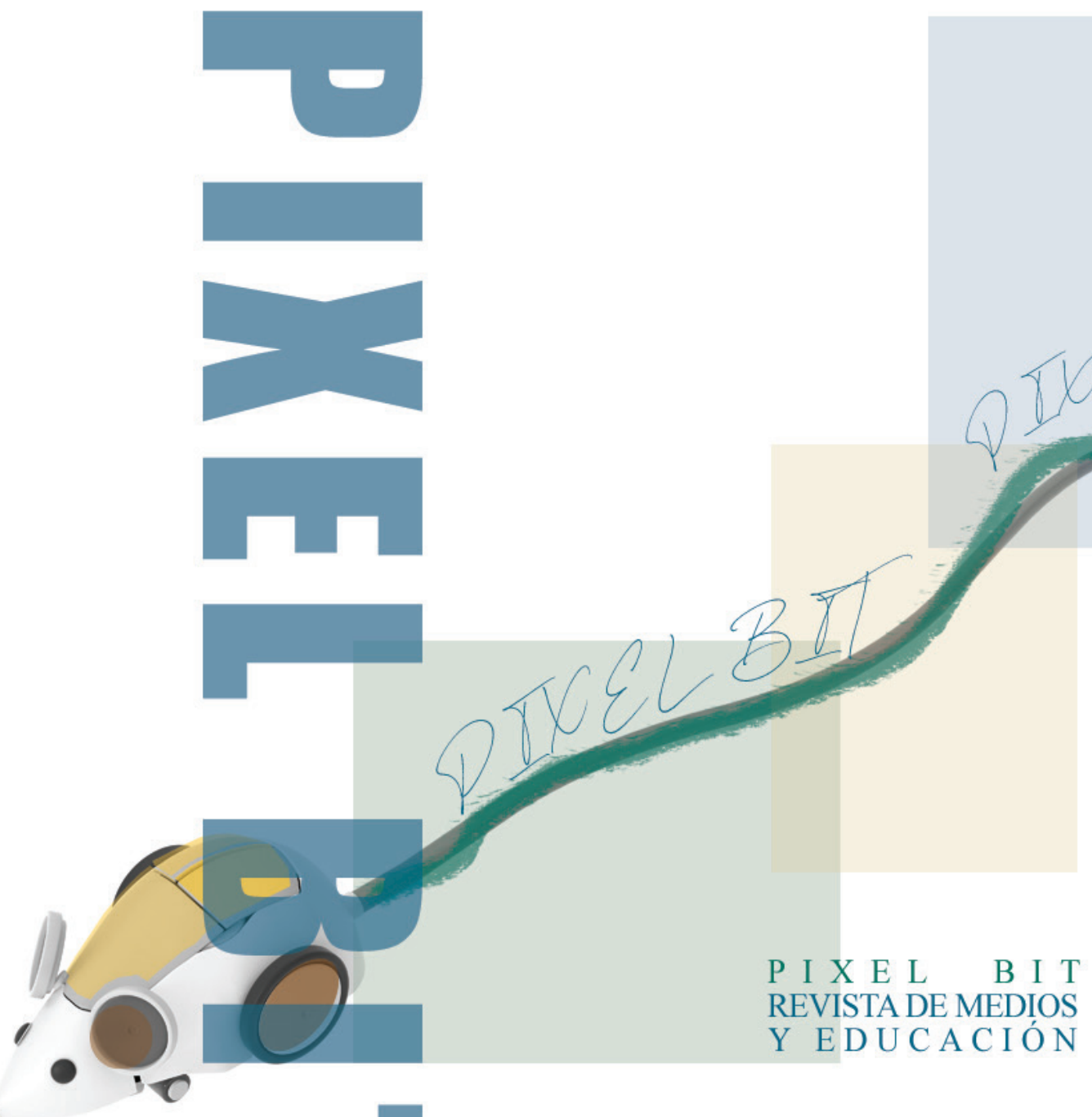


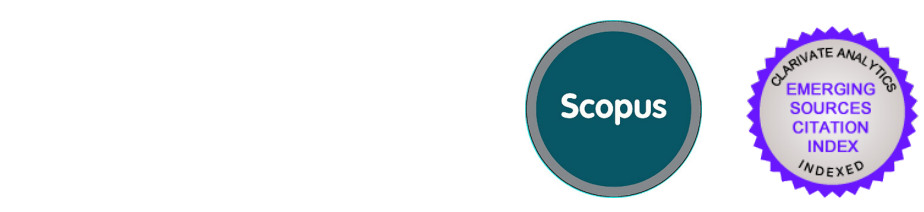

\section{PIXEL-BIT}

\section{REVISTA DE MEDIOS Y EDUCACIÓN}

N 58 - MAYO - 2020

https://recyt.fecyt.es/index.php/pixel/index 


\section{EQUIPO EDITORIAL (EDITORIAL BOARD)}

\section{EDITOR JEFE (EDITOR IN CHIEF)}

Dr. Julio Cabero Almenara, Departamento de Didáctica y Organización Educativa, Facultad de CC de la Educación, Universidad de Sevilla (España).

\section{EDITOR ADJUNTO (ASSISTANT EDITOR)}

Dr. Óscar M. Gallego Pérez, Secretariado de Recursos Audiovisuales y NN.TT., Universidad de Sevilla (España)

EDITOR EJECUTIVO/SECRETARIO GENERAL EDITORIAL (EXECUTIVE EDITOR)

Dr. Juan Jesús Gutiérrez Castillo, Departamento de Didáctica y Organización Educativa. Facultad de CC de la Educación, Universidad de Sevilla (España).

\section{CONSEJO DE REDACCIÓN}

\section{EDITOR}

Dr. Julio Cabero Almenara. Universidad de Sevilla (Espańa)

\section{EDITOR ASISTENTE}

Dr. Óscar M. Gallego Pérez. Universidad de Sevilla (España)

\section{SECRETARIO}

Dr. Juan Jesús Gutiérrez Castillo. Universidad de Sevilla (Espańa)

\section{VOCALES}

Dra. María Puig Gutiérrez, Universidad de Sevilla. (España)

Dra. Sandra Martínez Pérez, Universidad de Barcelona (Espańa)

Dr. Selín Carrasco, Universidad de La Punta (Argentina)

Dr. Jackson Collares, Universidades Federal do Amazonas (Brasil)

Dra. Kitty Gaona, Universidad Autónoma de Asunción (Paraguay)

Dra. Elvira Esther Navas, Universidad Metropolitana de Venezuela (Venezuela)

Dr. Angel Puentes Puente, Pontificia Universidad Católica Madre y Maestra. Santo Domingo (República Dominicana)

Dr. Fabrizio Manuel Sirignano, Università degli Studi Suor Orsola Benincasa (Italia)

\section{CONSEJO TÉCNICO}

Edición, maquetación: Manuel Serrano Hidalgo, Universidad de Sevilla (España)

Diseño de portada: Lucía Terrones García, S.A.V, Universidad de Sevilla (España)

Revisor/corrector de textos en inglés: Rubicelia Valencia Ortiz, MacMillan Education (México)

Revisores metodológicos: evaluadores asignados a cada artículo

Responsable de redes sociales: Manuel Serrano Hidalgo, Universidad de Sevilla (España)

Bases de datos: Bárbara Fernández Robles, Universidad de Sevilla (España)

Administración: Leticia Pinto Correa, S.A.V, Universidad de Sevilla (Espańa)

\section{CONSEJO CIENTÍFICO}

Jordi Adell Segura, Universidad Jaume I Castellón (España)

Ignacio Aguaded Gómez, Universidad de Huelva (Espańa)

María Victoria Aguiar Perera, Universidad de Las Palmas de Gran Canaria (España)

Olga María Alegre de la Rosa, Universidad de la Laguna Tenerife (Espańa)

Manuel Área Moreira, Universidad de la Laguna Tenerife (Espańa)

Patricia Ávila Muñoz, Instituto Latinoamericano de Comunicación Educativa (México)

Antonio Bartolomé Pina, Universidad de Barcelona (España)

Angel Manuel Bautista Valencia, Universidad Central de Panamá (Panamá)

Jos Beishuizen, Vrije Universiteit Amsterdam (Holanda)

Florentino Blázquez Entonado, Universidad de Extremadura (España)

Silvana Calaprice, Università degli studi di Bari (Italia)

Selín Carrasco, Universidad de La Punta (Argentina) 
Raimundo Carrasco Soto, Universidad de Durango (México)

Rafael Castañeda Barrena, Universidad de Sevilla (España)

Zulma Cataldi, Universidad de Buenos Aires (Argentina)

Manuel Cebrián de la Serna, Universidad de Málaga (Espańa)

Luciano Cecconi, Università degli Studi di Modena (Italia)

Jean-François Cerisier, Université de Poitiers, Francia

Jordi Lluís Coiduras Rodríguez, Universidad de Lleida (España)

Jackson Collares, Universidades Federal do Amazonas (Brasil)

Enricomaria Corbi, Università degli Studi Suor Orsola Benincasa (Italia)

Marialaura Cunzio, Università degli Studi Suor Orsola Benincasa (Italia)

Brigitte Denis, Université de Liège (Bélgica)

Floriana Falcinelli, Università degli Studi di Perugia (Italia)

Maria Cecilia Fonseca Sardi, Universidad Metropolitana de Venezuela (Venezuela)

Maribel Santos Miranda Pinto, Universidade do Minho (Portugal)

Kitty Gaona, Universidad Autónoma de Asunción (Paraguay)

María-Jesús Gallego-Arrufat, Universidad de Granada (España)

Fernando Gamboa Rodríguez, Universidad Nacional Autónoma de México

Lorenzo García Aretio, UNED (Espańa)

Ana García-Valcarcel Muńoz-Repiso, Universidad de Salamanca (España)

Antonio Bautista García-Vera, Universidad Complutense de Madrid (España)

José Manuel Gómez y Méndez, Universidad de Sevilla (España)

Mercedes González Sanmamed, Universidad de La Coruña (España)

Manuel González-Sicilia Llamas, Universidad Católica San Antonio-Murcia (España)

Ángel Pio González Soto, Universidad Rovira i Virgili, Tarragona (España)

António José Meneses Osório, Universidade do Minho (Portugal)

Carol Halal Orfali, Universidad Tecnológica de Chile INACAP (Chile)

Mauricio Hernández Ramírez, Universidad Autónoma de Tamaulipas (México)

Ana Landeta Etxeberría, Universidad a Distancia de Madrid (UDIMA)

Linda Lavelle, Plymouth Institute of Education (Inglaterra)

Fernando Leal Ríos, Universidad Autónoma de Tamaulipas (México)

Paul Lefrere, Cca (UK)

Carlos Marcelo García, Universidad de Sevilla (España)

Francois Marchessou, Universidad de Poittiers, París (Francia)

Francesca Marone, Università degli Studi di Napoli Federico II (Italia)

Francisco Martínez Sánchez, Universidad de Murcia (España)

Ivory de Lourdes Mogollón de Lugo, Universidad Central de Venezuela (Venezuela)

Angela Muschitiello, Università degli studi di Bari (Italia)

Margherita Musello, Università degli Studi Suor Orsola Benincasa (Italia)

Elvira Esther Navas, Universidad Metropolitana de Venezuela (Venezuela)

Trinidad Núńez Domínguez, Universidad de Sevilla (España)

James O’Higgins, de la Universidad de Dublín (UK)

José Antonio Ortega Carrillo, Universidad de Granada (Espańa)

Gabriela Padilla, Universidad Autónoma de Tumalipas (México)

Ramón Pérez Pérez, Universidad de Oviedo (España)

Angel Puentes Puente, Pontificia Universidad Católica Madre y Maestra. Santo Domingo (República Dominicana)

Julio Manuel Barroso Osuna, Universidad de Sevilla (España)

Rosalía Romero Tena. Universidad de Sevilla (España)

Hommy Rosario, Universidad de Carabobo (Venezuela)

Pier Giuseppe Rossi, Università di Macerata (Italia)

Jesús Salinas Ibáńez, Universidad Islas Baleares (España)

Yamile Sandoval Romero, Universidad de Santiago de Cali (Colombia)

Albert Sangrá Morer, Universidad Oberta de Catalunya (Espańa)

Ángel Sanmartín Alonso, Universidad de Valencia (España)

Horacio Santángelo, Universidad Tecnológica Nacional (Argentina)

Francisco Solá Cabrera, Universidad de Sevilla (España)

Jan Frick, Stavanger University (Noruega)

Karl Steffens, Universidad de Colonia (Alemania)

Seppo Tella, Helsinki University (Finlandia)

Hanne Wacher Kjaergaard, Aarhus University (Dinamarca) 


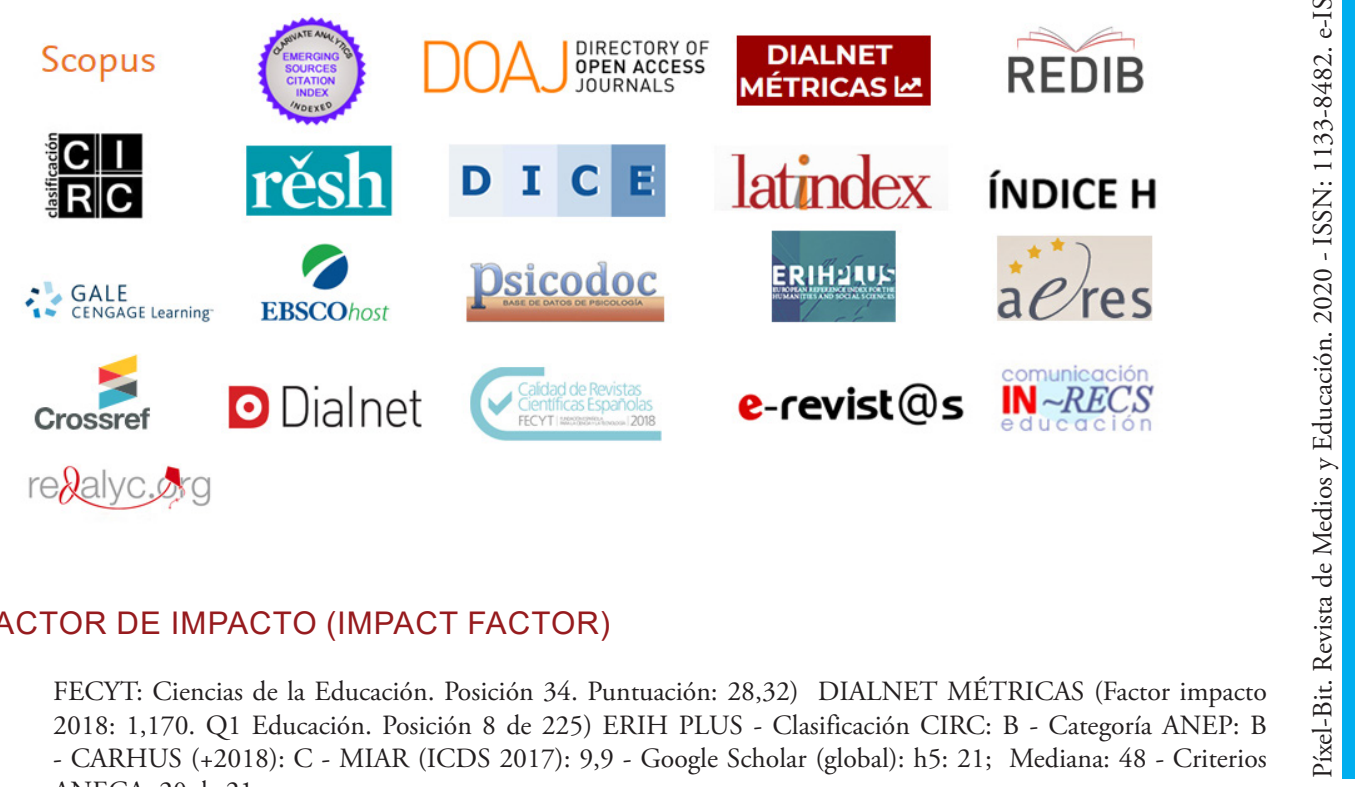

Píxel-Bit, Revista de Medios y Educación está indexada entre otras bases en: SCOPUS, Fecyt, Iresie, ISOC (CSIC/ CINDOC), DICE, MIAR, IN-RECS, RESH, Ulrich's Periodicals, Catálogo Latindex, Biné-EDUSOL, Dialnet, Redinet, OEI, DOCE, Scribd, Redalyc, Red Iberoamericana de Revistas de Comunicación y Cultura, Gage Cengage Learning, Centro de Documentación del Observatorio de la Infancia en Andalucía. Además de estar presente en portales especializados, Buscadores Científicos y Catálogos de Bibliotecas de reconocido prestigio, y pendiente de evaluación en otras bases de datos.

\section{EDITA (PUBLISHED BY)}

Grupo de Investigación Didáctica (HUM-390). Universidad de Sevilla (Espańa). Facultad de Ciencias de la Educación. Departamento de Didáctica y Organización Educativa. C/ Pirotecnia s/n, 41013 Sevilla.

Dirección de correo electrónico: revistapixelbit@us.es . URL: https://recyt.fecyt.es/index.php/pixel/index ISSN: 1133-8482; e-ISSN: 2171-7966; Depósito Legal: SE-1725-02

Formato de la revista: $16,5 \times 23,0 \mathrm{~cm}$

Los recursos incluidos en Píxel Bit están sujetos a una licencia Creative Commons Attribution-NonCommercialShareAlike 3.0 Unported (Reconocimiento-NoComercial-CompartirIgual)(CC BY-NC-SA 3.0), en consecuencia, las acciones, productos y utilidades derivadas de su utilización no podrán generar ningún tipo de lucro y la obra generada sólo podrá distribuirse bajo esta misma licencia. En las obras derivadas deberá, asimismo, hacerse referencia expresa a la fuente y al autor del recurso utilizado.

(C2020 Píxel-Bit. No está permitida la reproducción total o parcial por ningún medio de la versión impresa de la Revista Píxel- Bit. 
1.- Diseño de un instrumento de evaluación de aplicaciones digitales (Apps) que permiten desarrollar la competencia artística // Design of an instrument for evaluating digital applications (Apps) that allow students to develop artistic competence.

Martín Caeiro Rodríguez, Feliciano F. Ordoñez Fernández, María Dolores Callejón Chinchilla, Elke Castro León

2.- Nuevo método de análisis cualitativo mediante software para el análisis de redes sociales de la percepción grupal hacia las Matemáticas // New method of qualitative analysis using software for social networks analysis of group perception towards Mathematics.

Luis Manuel Soto-Ardila, Ana Caballero Carrasco, José Luis Carvalho, Luis Manuel Casas García

3.- Robótica y Proyectos STEAM: Desarrollo de la creatividad en las aulas de Educación Primaria // WhatsApp as an educational tool in Primary Education: students, teachers and families

Raquel Casado Fernández, Mirian Checa Romero

4.- WhatsApp como herramienta educativa en Educación Primaria: alumnado, docentes y familias // Methodologies \& Technologies to teach STEM in Primary Education: needs analysis Antonia Cascales-Martínez, Ma Ángeles Gomariz Vicente, Antonia Paco Simón

5.- Percepciones de los estudiantes sobre el uso de Facebook y Twitter en el contexto educativo por medio de la ciencia de datos y el aprendizaje automático // Perceptions of students about the use of Facebook and Twitter in the educational context through data science and machine learning

Ricardo-Adán Salas-Rueda

6.- Fortaleciendo el pensamiento computacional y habilidades sociales mediante actividades de aprendizaje con robótica educativa en niveles escolares iniciales // Strengthening computational thinking and social skills through learning activities with educational robotics in early school levels

Yen-Air Caballero-González, Ana García-Valcárcel Muñoz-Repiso

7.- Percepción de estudiantes de bachillerato sobre el uso de Metaverse en experiencias de aprendizaje de realidad aumentada en matemáticas // Perception of high school students about using Metaverse in augmented reality learning experiences in mathematics

Carlos Enrique George Reyes

8.- Tecnologías y cultura organizativa en los centros escolares. ¿La uberización de las relaciones laborales? // Technologies and organizational culture in schools. The labor relations' uberization?

M. Isabel Pardo Baldoví, Ángel San Martín Alonso

9.- University teachers' training: the Digital Competence // Formación del profesorado Universitario en la Competencia Digital (Bilingüe)

Adiela Ruiz Cabezas, María Castañar Medina Domínguez, Eufrasio Pérez Navío, Antonio María Medina Rivilla

10.- Alfabetización mediática en Educación Primaria. Perspectiva internacional del nivel de competencia mediática // Media Literacy in Primary Education. Internacional perspective of level of literacy competence

Rosa García-Ruiz, Armanda Pinto da Mota Matos, Arturo Arenas-Fernández, Cecilia Ugalde 


\title{
Robótica y Proyectos STEAM: Desarrollo de la creatividad en las aulas de Educación Primaria
}

\section{Robotics and STEAM projects: development of creativity in a Primary School Classroom}

\author{
Dra. Raquel Casado Fernández ${ }^{2}$ rcasado@formarobotik.com \\ Dra. Mirian Checa Romero' m.checa@uah.es \\ ${ }^{1}$ Universidad de Alcalá. Departamento de Ciencias de la Educación. Aulario María de Guzmán. Calle San Cirilo, s/n, \\ Alcalá de Henares, Madrid. (España). \\ ${ }^{2}$ FORMA ROBOTI-K S.L. Calle González Dávila, 18. Madrid. (España).
}

\section{RESUMEN}

La incorporación en las aulas de los proyectos STEAM y de la robótica es avalada por numerosos estudios que demuestran los efectos positivos en relación con la creatividad y la capacidad de resolver problemas, siendo estas habilidades necesarias para que los individuos puedan enfrentarse a los desafíos del siglo XXI. Este estudio pretende aportar evidencias empíricas sobre el aumento de la capacidad creativa en alumnos y alumnas de $5^{\circ}$ y $6^{\circ}$ de educación primaria. Para ello, se llevó a cabo un taller que se dividió en dos fases: la primera de ellas consistió en la incorporación en las aulas de los proyectos STEAM y la robótica como herramientas educativas analizando la variable creatividad a través del TestCREA, utilizando para ello un diseño pre-test/posttest; la segunda fase consistió en una competición donde el alumnado tuvo la ocasión de exponer sus proyectos STEAM y sus robot construidos y programados ante un comité de expertos, que evaluaron estos productos a través de los criterios de creatividad definidos por el mismo test. Los resultados obtenidos muestran un aumento significativo de la creatividad y una valoración muy positiva del comité de expertos, confirmando que los productos finales obtenidos por los estudiantes son altamente creativos.

\section{ABSTRACT}

The incorporation of STEAM (Science, Technology, Engineering, Arts and Mathematics) projects and robotics into the classroom is endorsed by numerous studies. Most of them demonstrate the positive effects in relation with creativity and resolving problems capability, being these abilities necessaries for students to face 21st century challenges. This study tries to bring up empiric evidences about the increase in creativity, through the analysis of the results from an educative workshop developed by 5 and 6th Primary Education. The workshop was divided in two phases: the first one was focused on incorporating STEAM projects and robotics in the classroom as an educative tools. These projects were incorporated in a transversal manner from a constructivist perspective and based on the scientific method. The impact on creativity was analysed through CREA test (using a pre-test and posttest). The second phase consisted in a contest where students presented their STEAM and robots projects. These products were assess by an expert committee using the creativity criteria defined in CREA test (newness, courage, truth and utility). Results show a significant increase on creativity measured through CREA test, and high scores on STEAM and robotics productions assessment, highlighting the opportunity to introduce these tools in primary school classrooms, to develop students' creativity.

\section{PALABRAS CLAVE}

STEAM; robótica educativa; creatividad; educación primaria; tecnología educativa.

\section{KEYWORDS}

STEAM; educative robotics; creativity; primary education; educative technology. 


\section{1.- Introducción}

Los cambios sociales y tecnológicos del siglo XXI plantean la necesidad de redefinir los modelos de enseńanza y aprendizaje para potenciar en los estudiantes el desarrollo de capacidades relacionadas con la creatividad y la resolución de problemas, unidas a la adquisición de competencias técnico-científicas que permitan resolver los retos inciertos del futuro.

Si partimos de un paradigma constructivista del aprendizaje, consideraremos que aprender significa transformar el conocimiento por parte del alumno. Para ello, deberemos diseñar escenarios de aprendizaje donde el alumno pueda implicarse en sus proyectos de aprendizaje. Si potenciamos Entornos de Aprendizaje Constructivistas EAC (Jonassen, 2008), fomentaremos en los estudiantes la resolución de problemas y su desarrollo conceptual. Los Modelos EAC son propuestas que parten de un problema, pregunta o proyecto como núcleo del entorno donde se ofrece al alumno varios sistemas de interpretación con el fin de resolver el problema y hallar las respuestas a las preguntas formuladas.

Uno de los ejemplos de estos Modelos EAC, podrían ser los proyectos STEAM, acrónimo de Sience, Technology, Engineering, Art and Mathematics, basado en el aprendizaje integrado de dichas disciplinas científicas, poniendo el foco principal en la resolución de problemas en situaciones abiertas y no estructuradas, con el fin de generar por parte del alumnado un determinado producto para solventar la situación problemática (Reinking \& Martin, 2018; Yakman \& Lee, 2012). Es por eso, que en estos casos añadimos una extensión del constructivismo denominado "Construccionismo" (Papert, 1980). Esta perspectiva defiende que el conocimiento es más efectivo cuando el estudiante está involucrado en la construcción de objetos que son significativos para él. En este sentido, se resalta la importancia del aspecto constructivo manual, fomentando así el pensamiento creativo y la construcción de nuevos conocimientos. Por otra parte, estos proyectos, muy ligados a la Robótica y Tecnología Educativa, resultan muy atractivos y motivadores al alumnado, además de que permiten desarrollar los objetivos, contenidos y competencias de las diferentes asignaturas (Arabit-García \& Prendes-Espinosa, 2020). La robótica educativa se presenta como un proceso creativo basado en el ensayo-error y como un proceso tecnológico basado en las interacciones entre sociedad, ciencia y tecnología, que se concretan en la programación, construcción y manipulación de una plataforma robótica (García, 2015; Karim, Lemaignan \& Mondada, 2015). En este sentido, la robótica educativa es una excelente herramienta educativa que, unida a una 
correcta planificación metodológica, podría mejorar significativamente el aprendizaje de los estudiantes, aumentar su motivación e interés, así como el desarrollo de habilidades de cooperación social, resolución de problemas y habilidades creativas.

Por tanto, la robótica educativa es una herramienta perfectamente integrable en un entorno de aprendizaje STEAM. La relación de los Proyectos STEAM con la tecnología y la ingeniería es muy estrecha y, como en estas disciplinas, el fin último es diseñar y llevar a la práctica una solución de un problema a través de la construcción de un objeto técnico, acercando estas disciplinas a priori alejadas, al currículo de la Enseñanza Primaria, adentrando a los alumnos en el denominado Método Científico (Suárez, García, Martínez \& Martos, 2018). Para ello, los nińos se adentran en un método de investigación, buscando diferentes caminos que guíen el descubrimiento, implicando necesariamente la puesta en práctica del pensamiento lógico. Dicha actividad científica necesita grandes dosis de imaginación y de una actitud crítica ante el problema a resolver para acercarse, de esta forma, a la construcción de conocimiento como si de científicos se tratasen. Incorporar estas herramientas en esta etapa educativa garantiza que los estudiantes sean capaces de utilizar el pensamiento científico a través de su propia experiencia. Por otro lado, también asegura el aspecto manipulativo con los objetos con los que están suficientemente familiarizados, ya que los estudiantes de los últimos cursos de esta etapa ( $5^{\circ}$ y $\left.6^{\circ}\right)$, tal y como nos indicaba Piaget (1978) se encuentran en la etapa de las operaciones concretas de su desarrollo. Se trata, por tanto, de una edad en la que empiezan a poder resolver problemas abiertos o semiabiertos planteados a través de elementos manipulativos, como es el caso de la robótica educativa y los proyectos STEAM (ej: Barker \& Ansorge, 2007).

Aunque la robótica educativa todavía no cuenta con suficientes investigaciones que demuestran una comprobación empírica suficiente, ya hay numerosos estudiosos que han analizado los cuantiosos beneficios que aportan, en las siguientes áreas: i) rendimiento académico: algunos estudios con metodologías cuantitativas han podido medir la mejora del rendimiento en los alumnos cuando utilizan robots en su aprendizaje (ej.: Barak \& Zadok, 2009; Kazakoff, Sullivan \& Bers, 2013; Toh. et. al, 2016); ii) interés y motivación: los estudios empíricos aportan datos acerca del interés que despiertan en los estudiantes el trabajo con robots (Mubin et al., 2012); iii) habilidades sociales y de trabajo cooperativo: las revisiones más recientes sobre el estudio de la robótica muestran conclusiones claras sobre los beneficios 
en estas áreas (Mitnik, Nussbaum \& Soto, 2008; Owens, Granader, Humphery \& Baron-Cohen, 2008). Varney et. al. (2012) muestran que la robótica es una herramienta eficaz para fomentar las habilidades de equipo en los estudiantes, ya que hace que discutan, resuelvan problemas de forma conjunta, trabajen con sus compañeros aportando cada uno lo mejor, generando experiencias de aprendizaje interactivas y atractivas para los niños que les impulsa a colaborar (Chang et al., 2010); iv) creatividad: es importante entender la creatividad como algo más que un factor individual, yendo más allá del genio solitario (Glăveanu, 2010). En la robótica educativa, la creatividad se asocia a los procesos de programación, construcción y manipulación de plataformas robóticas, que fomentan el pensamiento inventivo y la solución creativa de los problemas (Zawieska \& Duffy, 2015). En este sentido, existe un segundo foco basado en la creatividad entendida como la "ilusión de la vida" y la utilización de artefactos que realizan una serie de tareas que interactúan con el entorno. En el ámbito escolar, se ha confirmado que los estudiantes de Educación Primaria consiguen un aumento de la creatividad al trabajar con robots (Cavas et al., 2012) y, en relación a los proyectos STEAM, Puig y Bargalló (2017) aportó que arte y robótica forman un paradigma práctico que aporta belleza, alegría, diversión y creatividad mientras se aprenden contenidos curriculares; v) habilidades en la resolución de problemas: la robótica es una herramienta útil para la resolución de problemas (Benitti, 2012; Toh et al., 2016), aunque al analizar los resultados de algunos estudios encontramos algunas contradicciones. En los estudios de Williams, Ma, Prejean \& Ford (2007) no consiguieron resultados fiables, mientras que Sullivan (2008), por el contrario, encontró mejoras en las habilidades para resolver problemas al detectar un mayor uso de enfoques eficaces.

Por tanto, los proyectos STEAM aúnan dos de las principales metodologías del aprendizaje cooperativo, el aprendizaje basado en proyectos y el aprendizaje basado en problemas (Domingo \& Marqués, 2013). En base a una nueva pregunta o reto inicial, se plantea a los estudiantes generar un producto final que, para resolverlo, necesitarán superar diferentes tareas y pruebas, propios del aprendizaje basado en problemas y que genera altas dosis de aprendizaje significativo (Barak \& Assal, 2018). Ambas metodologías, por otra parte, llevan implícita la metodología del aprendizaje cooperativo (Domingo \& Marqués, 2013), que supondrá nuevas estructuras organizativas en el aula.

Con las previsiones y alcances anteriores, emerge la necesidad de formar a las nuevas generaciones en habilidades propias de la robótica y los proyectos STEAM para poder estar preparados para las futuras 
tecnologías que aún están por descubrir. Para ello, será necesario generar contextos innovadores de aprendizaje mucho más interdisciplinares, tecnológicos y creativos. En este sentido, la robótica educativa puede potenciar procesos creativos basados en el ensayo-error a través de procesos tecnológicos donde se conjugan la ciencia, la tecnología y la sociedad, que los estudiantes deberán concretar en la programación, construcción y manipulación de diferentes plataformas robóticas (García, 2015; Karim, Lemaignan \& Mondada, 2015). Por tanto, la robótica educativa se integra perfectamente en los entornos de aprendizaje STEAM, aportando cualidades como el interés, la motivación y el rendimiento de los estudiantes (Benitti, 2012; Alimisis, 2013; Mikropoulos \& Bellou, 2013).

El presente artículo tiene como objetivo examinar las mejoras experimentadas en la creatividad de los estudiantes de un aula de $5^{\circ}$ y $6^{\circ}$ de primaria, a través de la introducción de la robótica educativa y de los entornos de aprendizaje STEAM. Para ello, se analizan los resultados de un taller de 18 semanas de duración con 57 alumnos de entre 9 y 12 años pertenecientes a un colegio concertado de Móstoles, de la Comunidad de Madrid (España). Para valorar la creatividad se llevó a cabo un doble análisis: por un lado, un diseño cuasi experimental de grupo único con medidas repetidas pre-post-test a través del Test CREA de Inteligencia creativa y, por otro, el análisis de los productos STEAM elaborados por los estudiantes a través de los criterios establecidos por ese mismo Test.

El presente artículo se estructura de la siguiente forma: el epígrafe 2 presenta una descripción detallada de los materiales y métodos llevados a cabo para la realización del estudio, el epígrafe 3 presenta la metodología de análisis, así como los resultados obtenidos, el epígrafe 4 presenta la discusión y el epígrafe 5 las conclusiones.

\section{2.- Metodología}

La finalidad de este estudio fue doble: analizar y constatar mejoras en la creatividad a partir de un taller pedagógico que incorporaba la utilización de proyectos STEAM y robótica, utilizando para ello un diseño pre-test/post-test, con la que se llevó a cabo la evaluación antes y después del taller. Y, en segundo lugar, analizar los productos desarrollados por los estudiantes a través de una competición regulada por un comité de expertos. 


\subsection{Participantes}

En esta investigación participaron 57 alumnos que pertenecen a quinto y sexto de primaria de un colegio concertado en Móstoles (Madrid), con edades comprendidas entre los 9 y 12 años, de los cuales 27 eran de $5^{\circ}$ curso y 30 de $6^{\circ}$ curso. Del total de los estudiantes el $54 \%$ eran niños y el $46 \%$ eran niñas y fueron distribuidos a su vez en 9 grupos de 6-7 estudiantes, buscando la paridad entre las dos clases participantes. Por tanto, se formaron equipos mixtos de trabajo, de tal forma que los estudiantes de $6^{\circ}$ ejercieron el rol de "expertos" (puesto que ya era su segundo curso en el programa), mientras que los estudiantes de $5^{\circ}$ lo hicieron en el rol de "aprendices" (siendo su primer año en el programa). El profesorado de las asignaturas implicadas estaba presente en el aula, así como el equipo investigador que actuaba como observador participante. Los alumnos que forman parte del estudio fueron seleccionados de manera intencional en base al siguiente criterio: se seleccionó un centro en el que dentro de su proyecto educativo tuviera un programa transversal donde se aplicara la robótica y los proyectos STEAM como instrumentos de aprendizaje. Nuestra intervención se ajustó, por tanto, a la planificación del programa previamente diseñado por el centro. En este programa se respetaba la transversalidad curricular, trabajando contenidos curriculares de diferentes asignaturas implicadas, como la de Matemáticas, Ciencias Naturales, Lengua Española o Plástica.

\subsection{Materiales empleados en la intervención}

Para el desarrollo de esta investigación se utilizaron dos tipos de materiales: por un lado, los proyectos

STEAM y, por otro, el material del fabricante VEX IQ ${ }^{\circledR}$ para la programación y construcción de los robots. En relación a los proyectos STEAM, se optó por utilizarlos ya que se trata de proyectos transversales que utilizan la tecnología como nexo de unión, permitiendo incorporar conocimientos curriculares de las materias implicadas. Además, otra de sus características principales, es que posibilita el aprendizaje significativo, permitiendo crear artefactos que simulan la realidad, basándose para ello en la experimentación, lo que supone una herramienta especialmente interesante para utilizarla en el aula.

A través de este material, se planteó un reto al alumnado que, distribuido en grupos, debían de darle respuesta, siguiendo el método científico. Dada la naturaleza abierta de estos proyectos, no se impuso ninguna limitación en la utilización de múltiples recursos didácticos, ya que cada grupo podía incorporar aquellos que considerara necesarios para dar respuesta al reto planteado, desde los más tradicionales a los 
más actuales, como la robótica.

Como resultado de estos proyectos, se obtuvieron dos productos finales que posteriormente fueron evaluados: i) Cuaderno de campo: espacio donde el alumnado documentó todo el proceso creativo para dar respuesta al reto; ii) Maqueta: simulación de la idea que les permitió contrastar si realmente se estaba dando respuesta al reto planteado.

En relación a la construcción y programación de los robots, se utilizó el material proporcionado por VEX IQ (Figura 1), ya que está diseñado para el estudio de la robótica en educación primaria incorporando los cinco pilares básicos de STEAM. Además, este material permite trabajar en equipo en el aula, potenciando la capacidad de resolución de problemas y el liderazgo.

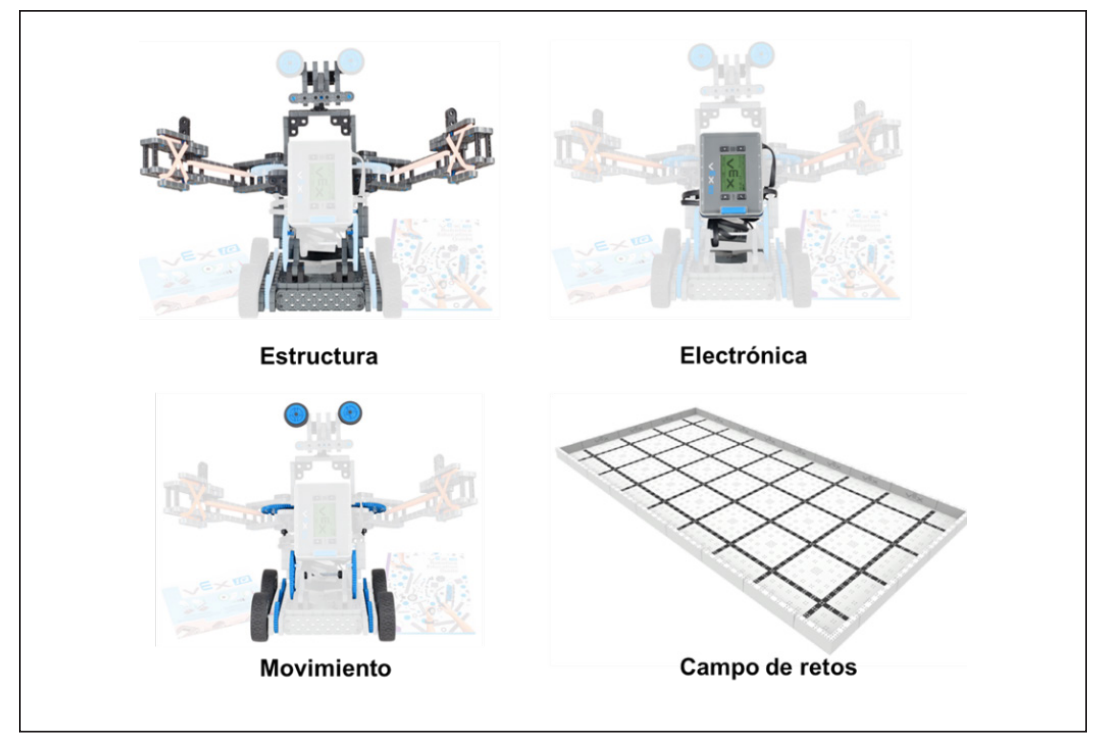

Figura 1. Material proporcionado por VEX IQ (Fuente: /www.vexrobotics.com)

Para el desarrollo de esta investigación se utilizaron dos materiales específicos: i) Kit de componentes: que incluyen las piezas necesarias para diseñar la estructura del robot, los mecánicos que permiten darle movimiento y la electrónica que facilita la comunicación y el control del robot mediante la programación del mismo; ii) Campo de retos: kit de 32 baldosas cuadradas que permite la creación de un campo de juego de $97 \times 49$ pulgadas ( $246 \times 124 \mathrm{~cm}$ - aprox), donde los robots se moverán según la programación desarrollada por los alumnos y alumnas para resolver los retos planteados. 
La combinación de estos materiales proporciona una oportunidad divertida, atractiva y práctica, para que los niños y niñas puedan explorar y experimentar por sí mismos, pudiendo dar rienda suelta a su imaginación y creatividad, creando así los robots libremente, con el objetivo de dar respuesta a los retos concretos planteados en el aula.

\subsection{Procedimiento}

La intervención fue llevada a cabo en 3 fases principales: planificación, desarrollo del programa y evaluación (Figura 2).

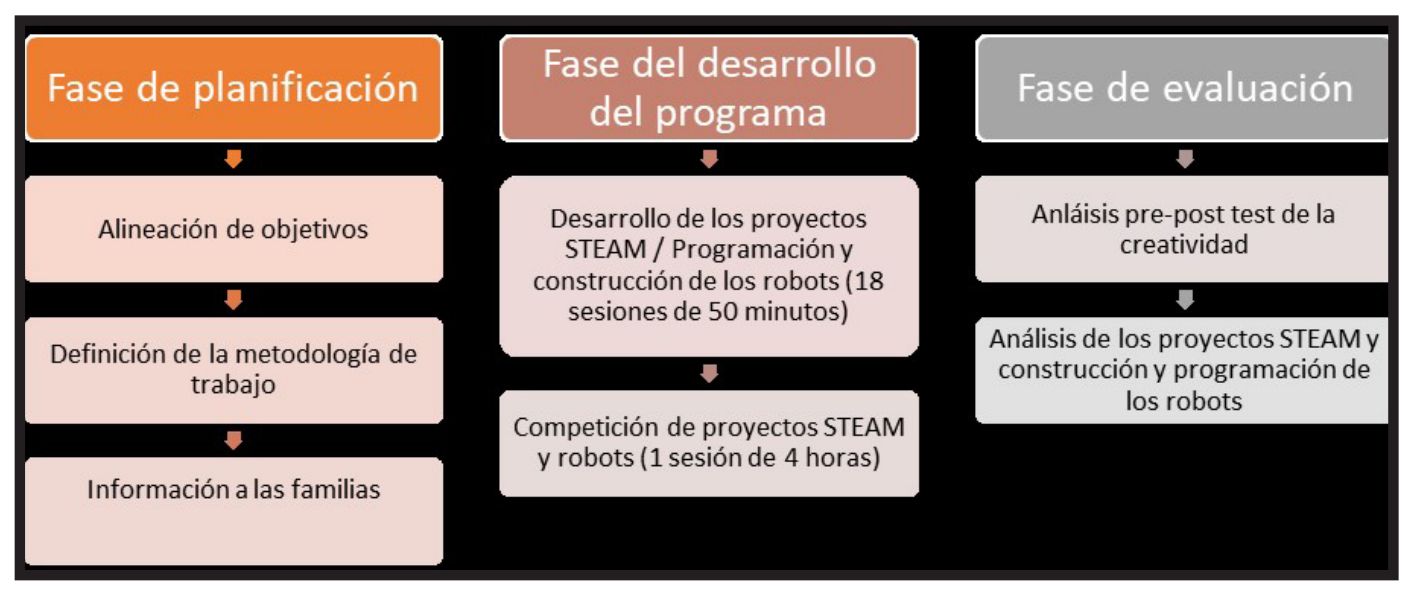

Figura 2. Fases del estudio (Fuente: Elaboración propia)

\subsubsection{Fase de planificación}

En esta fase se diferencian tres grandes acciones: La primera acción llevada a cabo fue conseguir la alineación de objetivos entre el profesorado y el equipo investigador a través de diversas reuniones, antes del inicio del curso escolar. La segunda acción consistió en definir el método de trabajo dentro del aula (reparto de equipos de trabajo, duración y fecha de las sesiones y equipo docente participante), así como los sistemas de recogida de datos (una cámara de video y una grabadora de audio por grupo de trabajo). La tercera y última acción que se llevó a cabo fue informar a las familias, para que conocieran de primera mano la existencia del equipo investigador en las aulas, y de cómo esta investigación afectaría al desarrollo de las clases. 


\subsubsection{Fase del Desarrollo del programa}

El programa estaba dividido, a su vez, en dos subfases: i) La primera de ellas consistió en un taller dividido en 18 sesiones de 50 minutos, en el que los estudiantes utilizaron tanto los proyectos STEAM como la construcción y programación de los robots; ii) la segunda subfase consistió en una competición donde cada equipo mostró su proyecto STEAM y las habilidades de su robot, esta competición se desarrolló en una única sesión de 4 horas.

Respecto a la primera subfase, el taller se ejecutó siguiendo la metodología científica, planteando dos retos que los estudiantes debían resolver. El primer reto se llevó a cabo a través de los proyectos STEAM, los estudiantes debían plantear una idea novedosa que permitiese ayudar en las tareas de la vida cotidiana, bajo el lema de "inventos para la comunidad". Estos retos dieron como resultado dos productos que posteriormente fueron evaluados: por un lado, el cuaderno de investigación y, por otro, una maqueta que ayudó a los estudiantes a materializar su idea. Ambos productos debían dar respuesta a los siguientes ítems:

- Tema de investigación: definición del invento de una manera concreta.

- Objetivo: definición más extensa de los beneficios que este invento supondría para la vida cotidiana.

- Hipótesis: unión del tema de investigación, el objetivo y el cómo se hará, en forma de pregunta.

- Estado de la cuestión: a través de la información en distintas fuentes (internet, libros y otros recursos), los niños y niñas argumentaban la viabilidad de su proyecto. Además, en aquellos proyectos que se utilizó la robótica, debían consultar a un experto para verificar su viabilidad.

- Plan de acción: debían hacer un guion indicando los pasos necesarios para desarrollar su idea en la vida real.

- Contrastar la hipótesis: mediante la construcción de una maqueta, los alumnos y alumnas comprobaron si su idea funcionaba.

- Conclusiones: los alumnos y alumnas documentaron los resultados obtenidos tras las comprobaciones realizadas con la ayuda de la maqueta. 
El segundo reto planteado consistió en que los robots construidos y programados, pudieran desplazar objetivos esféricos, pasando por un puente que unía las dos partes del campo de juego. Para dar respuesta a este segundo reto, los alumnos y alumnas siguieron los siguientes pasos:

- Creación de los robots: los niños y niñas distribuidos por grupos decidieron cómo crear el robot para que cumpliese con el reto planteado.

- Programación de los robots: donde los alumnos diseñaron los pasos que debían seguir los robots para que pudiesen cumplir los retos.

- Testeo de la solución: los alumnos hicieron distintas pruebas en el campo de juego para comprobar si la construcción y programación del robot era la adecuada. Si no fuera así, debían hacer los ajustes necesarios, tanto en la creación como en la programación.

En cuanto a la segunda subfase y como cierre del programa, el centro educativo realizó una competición en la que participaron tanto los estudiantes participantes en la intervención, como un jurado compuesto por miembros del equipo docente, que ejerció como comité de expertos. En dicha competición, cada equipo presentó de forma libre su proyecto STEAM, pudiendo optar por diferentes métodos, además de las habilidades de su robot, ya que cada equipo intentó resolver los retos planteados por el comité de expertos, tal y como puede verse en la figura 3.

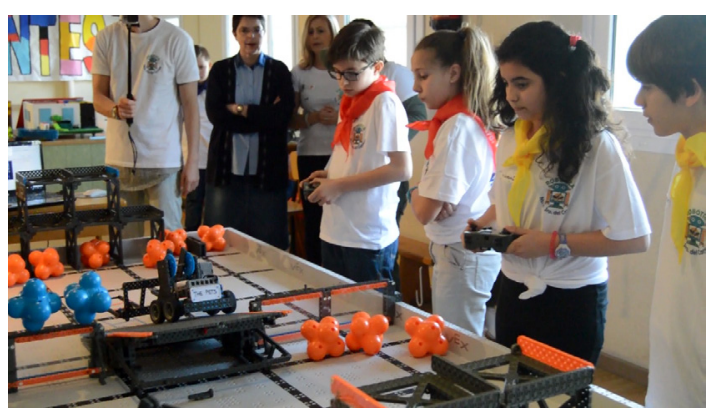

Figura 3. Competición de los proyectos STEAM (Fuente: Elaboración propia)

\subsubsection{Fase de evaluación}

El análisis de los resultados del taller se ha realizado desde un doble enfoque: el análisis de la variable de creatividad 
a través del Test CREA, y el análisis de los proyectos STEAM y la programación de los robots creados por los estudiantes. El análisis de los datos se ha realizado con el programa Microsoft Excel.

Para al análisis de la creatividad de los alumnos, se utilizó el Test CREA de Inteligencia Creativa (Corbalán et al., 2003), a través de un diseño pre-test/post-test. Dicho test valora la capacidad creativa a través de una evaluación cognitiva de la creatividad individual según el indicador de generación de cuestiones en el contexto teórico de búsqueda y solución de problemas. La aplicación de la prueba consiste en la presentación de 3 láminas ante las que los alumnos tienen que hacerse el mayor número de preguntas posible. La prueba tiene un coeficiente de fiabilidad Alpha de Cronbach de 0,775.

En lo que respecta a la evaluación de los proyectos STEAM y la programación de los robot construidos, se emplearon los criterios propuestos por el mismo test para la valoración de productos creativos: el criterio de novedad, entendido como lo que es estadísticamente raro o no frecuente, es decir, cuando aparece por primera vez en el tiempo; el criterio de valor, que se refiere al valor que tiene el producto creado en relación a la asignatura; el criterio de verdad, que se aplicaría a la valoración de ideas en un sistema lógico donde puede darse lo verdadero y lo falso, pero no podría aplicarse en sistemas no lógicos y el criterio de utilidad, que se refiere a la efectividad de predicción, es decir, indica lo que va a suceder si se dan unas condiciones determinadas. En definitiva, se considera que para que una idea sea creativa no sólo debe ser nueva, sino también valiosa, verdadera y útil. Para ello, los proyectos generados por los estudiantes fueron calificados por un comité de expertos extraídos de los profesores de las asignaturas implicadas, mediante la asignación de un valor numérico en una escala de 1 a 10 para cada uno de los criterios anteriores.

\section{3.- Análisis y resultados}

\subsection{Comparación pre-test/post-test de la creatividad de los estudiantes}

Para el análisis estadístico de los resultados se realizó la prueba de Kolmogorov-Smirnov (p=0,02), que puso de manifiesto que la variable creatividad se distribuía normalmente, por lo que, para la comparación de los resultados del pre y post-test, se decidió el empleo de la prueba paramétrica de contraste de medias para datos apareados para comprobar si existían diferencias significativas en creatividad antes y después de la participación en el programa. Además, se comprobó el supuesto de homocedasticidad de varianzas, realizándose posteriormente un ANOVA con los datos del pre-test $(\mathrm{F}=2,6325 ; \mathrm{p}=0,05475)$ rechazándose 
la hipótesis de diferencias de partida entre ellas, siendo este procedimiento una garantía de control.

Para los valores de la creatividad obtenidos en el pre-test y post-test el contraste de medias reveló diferencias estadísticamente significativas entre los resultados en todos los participantes (Lámina A: t= $-3,4965 \mathrm{p}=0,0005$ y Lámina C: $\mathrm{t}=-2,0151 \mathrm{p}=0,0248)$. También reveló diferencias estadísticamente significativas entre los resultados individualmente de los estudiantes de $5^{\circ}$ curso, primer año en el programa (Lámina A: $\mathrm{t}=-2,5688$ p=0,0082 y Lámina C: $\mathrm{t}=-1,3907 \mathrm{p}=0,0882$ ) y los de $\sigma^{\circ}$ curso, segundo año en el programa (Lámina A: $t=-2,3380 p=0,0149$ y Lámina $C: t=-1,4313 p=0,0838$ ). En la figura 4 se muestra los resultados en creatividad de los nińos y niñas en el pre y post-test CREA antes y después de la participación en el programa. Igualmente, en la tabla 1 se muestran los estadísticos descriptivos de cada una de las clases en el pre y en el post-test.

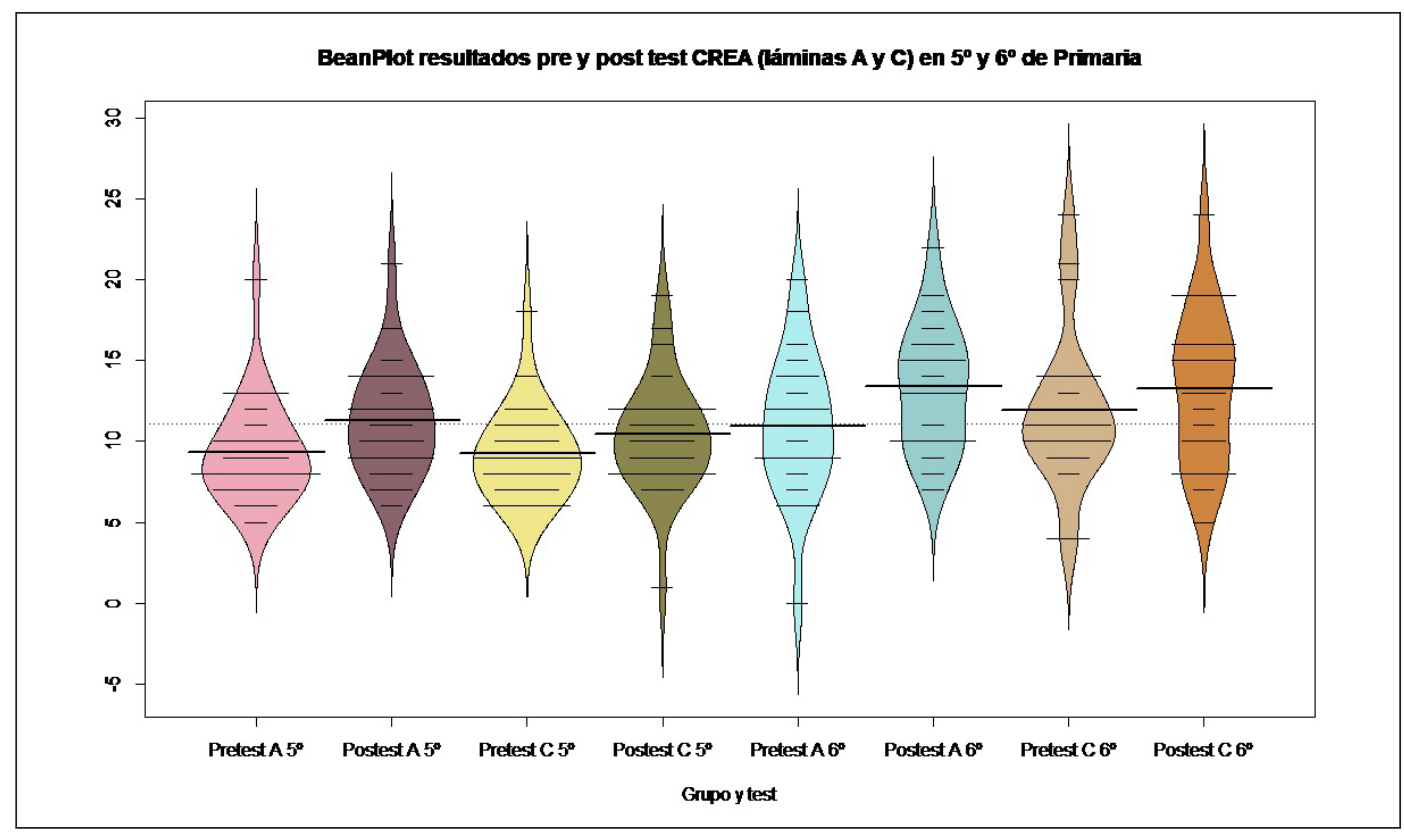

Figura 4. BeanPlot de la variable creatividad pre y post test de los alumnos de $5^{\circ}$ y $6^{\circ}$ de Primaria

Los resultados obtenidos confirman que la incorporación de los proyectos STEAM y la robótica a través de un taller educativo en las asignaturas implicadas, suponen un aumento de la creatividad en el alumnado. Los estudiantes participantes en esta investigación han mejorado su creatividad en la aplicación post-test del Test CREA. Por tanto, se puede afirmar que la participación en el proyecto fue eficaz para incrementar 
la creatividad, tanto de forma global como en cada uno de los cursos participantes por separado.

Tabla 1. Estadísticos descriptivos de la variable creatividad en los dos grupos pre y post-test

\begin{tabular}{|l|c|c|c|c|c|c|}
\hline \multirow{2}{*}{} & \multicolumn{2}{|c|}{ Total } & \multicolumn{2}{c|}{ Robótica 10 ańo } & \multicolumn{2}{c|}{ Robótica 2o ańo } \\
\cline { 2 - 7 } & LamA & LamC & LamA & LamC & LamA & LamC \\
\hline $\begin{array}{l}\text { Promedio } \\
\text { pre-test }\end{array}$ & 10,043 & 10,447 & 9,038 & 9,269 & 10,952 & 11,905 \\
\hline $\begin{array}{l}\text { Promedio } \\
\text { post-test }\end{array}$ & 12,234 & 11,702 & 11,308 & 10,423 & 13,381 & 13,286 \\
\hline & $21,82 \%$ & $12,02 \%$ & $21,49 \%$ & $12,45 \%$ & $22,17 \%$ & $11,60 \%$ \\
\hline
\end{tabular}

\subsection{Resultados de la competición de la robótica y los proyectos STEAM}

Para la evaluación emitida por el comité de expertos se utilizaron los criterios de creatividad definidos por el mismo test CREA (novedad, valor, verdad y utilidad). Dado que durante el programa los estudiantes trabajaron en grupo, la evaluación no se realiza a modo individual. La tabla 2 muestra las valoraciones para cada equipo, realizadas por el comité de expertos, sobre la creatividad de los productos realizados. Se observa, en general, que fueron muy bien puntuadas con valores promedio a partir de 7 (novedad: 7; valor: 8 ; verdad: 8,17 y utilidad: 7,17$)$.

Tabla 2. Valoraciones de los proyectos STEAM y robótica por el comité de expertos

\begin{tabular}{|c|c|c|c|c|}
\hline Cabecera & Novedad & Valor & Verdad & Utilidad \\
\hline Grupo 1 & 6,00 & 8,00 & 7,00 & 6,00 \\
\hline Grupo 2 & 5,00 & 8,00 & 8,00 & 8,00 \\
\hline Grupo 3 & 8,00 & 8,00 & 8,00 & 6,00 \\
\hline Grupo 4 & 9,00 & 8,00 & 6,00 & 7,00 \\
\hline Grupo 5 & 7,00 & 8,00 & 10,00 & 9,00 \\
\hline Grupo 6 & 7,00 & 8,00 & 10,00 & 7,00 \\
\hline Promedio & 7,00 & 8,00 & 8,17 & 7,17 \\
\hline
\end{tabular}

Si analizamos los proyectos anteriores, los resultados muestran que el producto mejor valorado fue el del grupo 5 (novedad: 7, valor: 8, verdad: 10 y utilidad: 9) y el peor valorado fue el grupo 1 (novedad: 6, valor: 8, verdad: 7 y utilidad: 6). Se observa que todos los grupos tienen una valoración media en torno a 7 en una escala de 0 a 10, siendo la valoración media grupal de 7,58, por lo que se confirma que los productos creados por los estudiantes son altamente creativos. 


\section{4.- Discusión}

Numerosas investigaciones concluyen resultados semejantes a los encontrados en el presente estudio, estableciendo una relación estrecha entre el uso de los proyectos STEAM y la robótica educativa con la creatividad o el pensamiento creativo. De acuerdo con la concepción de creatividad de algunos autores, esta se entiende como la capacidad de producir respuestas o soluciones originales para los problemas (Waisburd 2004). Sin embargo, dichas respuestas requieren que sean adoptadas y reconocidas por las organizaciones o entornos donde se producen. Por tanto, una idea, por más original que resulte, no será útil si no se puede llevar a la práctica con éxito (Acuña Zúniga, 2012). Por esta razón, es en las escuelas y contextos educativos donde se debe favorecer el pensamiento creativo, para que los estudiantes otorguen sentido a la realidad donde se desarrollan.

En el marco de nuestra experiencia, hemos concebido la robótica educativa y los proyectos STEAM como un contexto de aprendizaje que promueve un conjunto de desempeńos y habilidades directamente relacionados con la creatividad (Aris \& Orcos, 2019). Gracias a las creaciones desarrolladas por los estudiantes, han recreado su cotidianeidad a través de simulaciones para resolver problemas en un tiempo y en un espacio determinado para facilitar las formas de vida. En este sentido, han desarrollado la habilidad de aplicar las tecnologías digitales a diferentes situaciones más o menos complejas, tratando de adelantar las consecuencias que de éstas se derivan. A este respecto, García (2015) analizó la robótica como parte del proceso educativo, entendiendo que programar va más allá del acto de codificar, ya que conforma un conjunto de acciones que giran en torno a cuatro palabras clave: imaginar, diseńar, construir y programar. Kim \& Chae (2016), por su parte, en su estudio sobre la aplicación y desarrollo de los proyectos STEAM, mantienen que la convergencia de las ciencias, tecnología, ingeniería, arte y matemáticas mejora las habilidades creativas necesarias para la resolución de problemas. En este mismo sentido, en los estudios desarrollados por Alimisis (2013) afirma que los robots desempeñan un papel positivo en el aprendizaje de actividades educativas, poniendo el foco en el pensamiento creativo y en las mejoras de las habilidades para resolver problemas.

Por todo esto, tanto la robótica educativa como los proyectos STEAM pueden adoptar el rol de herramientas educativas para fomentar la búsqueda de soluciones creativas a problemas inciertos, con el fin de convertirse en experiencias simuladas para resolver los diferentes retos del futuro que les planteará 
la sociedad. En este sentido, permiten proporcionar espacios lúdicos que se asemejan a situaciones y escenarios del mundo real, donde deben buscar soluciones complejas, sirviendo de ensayo continuo para las exigencias futuras.

En cuanto a la metodología empleada en este estudio, ha permitido conocer y analizar el punto de partida y final de la creatividad (Rebetez \& Betrancourt, 2007), así como las valoraciones de los productos obtenidos por parte del comité de expertos. Esta metodología se completará, en investigaciones sucesivas, con un estudio etnográfico (Hine, 2005), donde se analice en profundidad los discursos de los estudiantes para comprender el proceso llevado a cabo para la obtención de los resultados alcanzados. Además, aunque el aumento de la creatividad ha sido significativo en cada uno de los grupos, quizá se podría enriquecer el estudio con el empleo de un grupo control para eliminar el efecto del aumento de la creatividad del alumnado como consecuencia de la sobreexposición al test CREA.

\section{5.- Conclusiones}

Con respecto al objetivo general del estudio, se concluye que la incorporación de las herramientas educativas como la robótica y los proyectos STEAM en las aulas de educación primaria, resulta muy beneficiosa para los estudiantes, ya que favorece su capacidad creativa para la resolución de problemas. Sin embargo, la robótica o los proyectos STEAM, por sí solos, no pueden mejorar el aprendizaje ni las habilidades analizadas. Para ello, es fundamental la utilización que se haga de ellos en las escuelas. Por este motivo, se considera necesario llevar a cabo nuevas investigaciones que permitan profundizar en el conocimiento de estas poderosas herramientas.

No obstante, debemos ser conscientes de que esto implica un gran reto, tanto para los docentes como para las instituciones educativas en general. Los docentes deben prepararse tecnológicamente para llevar a cabo tanto proyectos STEAM en general como de robótica educativa en concreto, tratando de potenciar el método científico. De esta forma, tal y como ocurre en el presente estudio, se podrán plantear metodologías que pongan el foco en el desarrollo de las habilidades cognitivas analizadas, así como la incorporación y profundización en los conocimientos curriculares de las materias implicadas.

Todo esto, supondrá la necesidad de que docentes e instituciones salgan de su zona de confort para 
enfrentarse a este nuevo desafío, además de salvar la difícil barrera de la limitación de tiempo impuesta por el sistema educativo actual. Por tanto, utilizar las herramientas mencionadas se convierte en un desafío para las comunidades educativas para que busquen los recursos y el apoyo de las administraciones y que estas metodologías sean validadas para que sean implementadas como una práctica regular en los diferentes ambientes de aprendizaje. No obstante, si el objetivo de la educación es formar a ciudadanos preparados para el futuro, la aplicación de estas herramientas en las aulas debe convertirse en una realidad.

\section{Referencias bibliográficas}

Acuña Zúñiga, A. L. (2012). Diseño y administración de proyectos de robótica educativa: lecciones aprendidas. Teoría de la Educación. Educación y Cultura en la Sociedad de la Información, 13(3), 6-27. Recuperado de https://bit.ly/2OUUDBU

Alimisis, D. (2013) Educational robotics: open questions and new challenges. Themes in science \& technology education, 6(1), 63-71. Recuperado de https://bit.ly/2rtKPXq

Arabit-García J. \& Prendes-Espinosa, Ma . P. (2020). Metodologías y Tecnologías para enseñar STEM en Educación Primaria: análisis de necesidades. Pixel-Bit. Revista de Medios y Educación, 57. https://doi.org/10.12795/pixelbit.2020.i57.04

Aris, N. \& Orcos, L. (2019). Educational Robotics in the Stage of Secondary Education: Empirical Study on Motivation and STEM Skills. Education Sciences, 9(73), 1-15. DOI: https://bit.ly/37HK6SS

Barak, M. \& Assal, M. (2018). Robotics and STEM learning: students' achievements in assignments according to the P3 Task Taxonomy-practice, problem solving, and projects. International Journal of Technology and Design Education, 28(1), 121-144.

http://dx.doi.org/10.1007/s10798-016-9385-9

Barak, M. \& Zadok, Y. (2009). Robotics projects and learning concepts in science, technology, and problem solving. International Journal of Technology \& Design Education, 19(3), 289-307. https://doi.org/10.1007/s10798-007-9043-3

Barker, B. \& Ansorge, J. (2007). Robotics as Means to Increase Achievement Scores in an Informal Learning Environment. Journal of research on technology in education, 39(3), 229-243.

https://doi.org/10.1080/15391523.2007.10782481

Píxel-Bit. Revista de Medios y Educación. 2020 - ISSN: 1133-8482. e-ISSN: 2171-7966. 
Benitti, V. (2012). Exploring the educational potential of robotics in schools: A systematic review. Computers \& Education, 58(3), 978-988. https://doi.org/10.1016/j.compedu.2011.10.006

Cavas, B., Kesercioglu, T., Holbrook, J., Rannikmae, M., Ozdogru, E. \& Gokler, F. (2012). The effects of robotics club on the students' performance on science process \& scientific creativity skills and perceptions on robots, human and society. 3rd International Workshop teaching robotics, teaching with robotics integrating robotics in school curriculum (pp. 40-50). Riva del Garda, Italy. Recuperado de https://bit.ly/2OmGXQO

Chang, C., Lee, J., Chao, P., Wang, C. \& Chen, G. (2010). Exploring the possibility of using humanoid robots as instructional tools for teaching a second language in primary school. Educational Technology \& Society, 13(2), 13-24. Recuperado de https://bit.ly/2QV8smh

Corbalán, J., Martínez, F., Donolo, D., Alonso, C., Tejerina, M., \& Limiñana, M. R. (2003). CREA. Creative intelligence, a cognitive measure of creativity. Madrid: TEA Editions.

Domingo, M. \& Marqués, P. (2013). Práctica docente en aulas 2.0 de centros de educación primaria y secundaria de España. Píxel-Bit. Revista medios y educación, 42(1), 115-128.

Recuperado de https://bit.ly/33khVpO

García, J. M. (2015). Robótica educativa. La programación como parte de un proceso educativo. REDRevista de Educación a Distancia, 46(8), 1-11.

Glăveanu, V. (2010). Paradigms in the study of creativity: introducing the perspective of cultural psychology. New ideas in psychology, 28(1), 79-93. http://doi.org/10.1016/j.newideapsych.2009.07.007

Hine, C. (2005). Virtual Methods. Issues in social research on the Internet. New York: Berg Publishers. https://doi.org/10.1108/00242530710831338

Jonassen, D. (2008). Instructional design as design problem solving: An iterative process. Educational technology: The magazine for managers of change in education, 48(3), 21-26.

Recuperado de https://bit.ly/2XTjWbC

Karim, M., Lemaignan, S., \& Mondada, F. (2015). A Review: Can Robots Reshape K-12 STEM Education? In Advanced Robotics and its Social Impacts (ARSO). IEEE International Workshop on, (1-8). https://doi.org/10.1109/ARSO.2015.7428217

Kazakoff, E., Sullivan, A., \& Bers, M. (2013). The effect of a classroom-based intensive robotics and programming workshop on sequencing ability in early childhood. Early Childhood Education Journal, 
41(4), 245-255. https://doi.org/10.1007/s10643-012-0554-5

Kim, H., \& Chae, D. (2016). The development and application of a STEAM program based on traditional Korean culture. Eurasia Journal of Mathematics, Science and Technology Education, 12(7), 1925-1936. https://doi.org/10.12973/eurasia.2016.1539a

Mikropoulos, T. \& Bellou, I. (2013). Educational Robotics as Mindtools. Themes in Science \& Technology Education, 6(1), 5-14. Recuperado de https://bit.ly/2Y1gWtP

Mitnik, R., Nussbaum, M. \& Soto, A. (2008). An autonomous educational mobile robot mediator. Department of computer science. Autonomous Robots, 25(4), 367-382. https://doi.org/10.1007/ s10514-008-9101-z

Mubin, O., Bartneck, C., Feijs, L., Hooft van Huysduynen, H., Hu, J. \& Muelver, J. (2012). Improving speech recognition with the robot interaction language. Disruptive science and Technology, 1(2), 79-88. https://doi.org/10.1089/dst.2012.0010

Owens, G., Granader, Y., Humphrey, A., \& Baron-Cohen, S. (2008). LEGO Therapy and the social use of language programme: an evaluation of two social skills interventions for children with high functioning autism and asperger sindrome. Journal of Autism and Developmental Disorder, 38, 19441957. https://doi.org/10.1007/s10803-008-0590-6

Papert, S. (1980). Mindstorms: Children, computers, and powerful ideas. New York: Basic Books. Recuperado de https://bit.ly/2OOKeHH

Puig, N. \& Bargalló, C. (2017). Aprendizaje de las ciencias basado en proyectos: del contexto a la acción. Ápice. Revista de Educación Cientifica, 18(1), 3-16. https://doi.org/10.17979/arec.2017.1.1.2020

Piaget, J. (1978). La equilibración de las estructuras cognitivas. Problema central de desarrollo. Madrid: Siglo XXI.

Rebetez, C. \& Betrancourt, M. (2007). Video game research in cognitive and educational sciences. Cognitive Creier Comportament, 11(1), 131-142

Reinking, A. \& Martin, B. (2018). The gender gap in STEM fields: Theories, movements, and ideas to engage girls in STEM. Journal of New Approaches in Educational Research, 7(2), 148-153. https://doi.org/10.7821/naer.2018.7.271

Suárez, A., García, D., Martínez, P. \& Martos, J. (2018). Contribución de la robótica educativa en la adquisición de conocimientos de matemáticas en la Educación Primaria. Magister, 30(1,2), 43-54. 
https://doi.org/10.17811/msg.30.1.2018.43-54

Sullivan, F. (2008). Robotics and science literacy: thinking skills, Ssience. Process skills and systems understanding. Journal of research in science teaching, 45(3), 373-394. https://doi.org/10.1002/tea.20238

Toh, L. P. E., Causo, A., Tzuo, P. W., Chen, I. M. \& Yeo, S. H. (2016). A Review on the Use of Robots in Education and Young Children. Educational Technology \& Society, 19(2), 148-163. Recuperado de https://bit.ly/2Dm0vi3

Varney, M. W., Janoudi, A., Aslam, D. M. y Graham, D. (2012). Building young engineers: TASEM for third graders in woodcreek magnet elementary school. IEEE Transactions on education, 55(1), 78-82. https://doi.org/10.1109/TE.2011.2131143

Waisburd, G. (2004). El poder de tu creatividad. México: American Book Store.

Williams, D., Ma, Y., Prejean, L. \& Ford, M.J. (2007). Acquisition of Physics Content Knowledge and Scientific Inquiry Skills in a Robotics Summer Camp. Journal of Research on Technology in Education, 40(2), 201-216. https://doi.org/10.1080/15391523.2007.10782505

Yakman, G. \& Lee, L. (2012). Exploring the Exemplary STEAM Education in the U.S. as a Practical Educational Framework for Korea. Journal of the Korean Association for Research in Science Education, 32(6), 1072-1086. https://doi.org/10.14697/jkase.2012.32.6.1072

Zawieska, K., \& Duffy, B. (2015). The social construction of creativity in educational robotics. In R. Szewczyk et al. (Eds.), Progress in Automation, Robotics and Measuring Techniques. Advances in Intelligent Systems and Computing, vol 351. Springer, 329-338.

\section{Cómo citar este artículo:}

Casado, R. \& Checa-Romero, M. (2020). Robótica y Proyectos STEAM: Desarrollo de la creatividad en las aulas de Educación Primaria Pixel-Bit. Revista de Medios y Educación. 58, 51-69. https://doi.org/10.12795/pixelbit.73672 